Revista de

Contabilidade e

Organizações

www.rco.usp.br
DOI: http://dx.doi.org/10.11606/issn.1982-6486.rco.2018.142273
Journal of

Accounting and

Organizations

www.rco.usp.br

\title{
Reflexos da cultura local nos sistemas de controle de incubadoras de empresas
}

Reflexes of local culture on the business incubators' control systems

Iago Franca Lopes ${ }^{\text {a }}$ Ilse Maria Beuren ${ }^{\mathrm{a}}$

${ }^{a}$ Universidade Federal de Santa Catarina

Palavras-chave

Condições culturais

Alavancas de controle.

Incubadoras de empresas.

\begin{abstract}
Resumo
Esta pesquisa tem por objetivo analisar o reflexo das condições culturais locais no uso das 'alavancas de controle' no ambiente de inovação tecnológica das incubadoras de empresas. A amostra da survey compõe-se de 75 gestores de incubadoras de empresas. Os resultados da modelagem de equações estruturais mostram que uma maior incorporação das condições culturais locais nas incubadoras se reflete no uso das alavancas de controle presentes. Especificamente, observou-se que a permeabilidade a estas condições culturais presentes no ambiente externo favorecem a adequação das alavancas ao contexto. O estudo fornece implicações práticas para o campo das incubadoras de empresas, e alerta os gestores sobre a relevância em considerar as condições culturais locais na adaptação e no uso das alavancas de controle.
\end{abstract}

Keywords

Cultural conditions.

Levers of control.

Business Incubators.
Informações do Artigo

Recebido: 10 de janeiro de 2018

Aceito: 19 de julho de 2018

Publicado: 24 de julho de 2018

\begin{abstract}
This research aims to analyze the reflexes of the local cultural conditions on the usage of the 'levers of control' by business incubators in an environment of technological innovation. The survey sample consists of 75 managers of business incubators. The results of the structural equations modeling show that the higher acceptance of local cultural conditions by business incubators is reflected on the usage of the levers of control' adopted by these organizations. Specifically, we observed that whether the incubator is receptive for local cultural conditions from the external context the better is the controls'fit to the context itself. The study provides practical implications for the field of business incubators and alerts managers about the relevance of considering local cultural conditions to adapt and use the 'levers of control'.
\end{abstract}

\section{Implicações práticas}

O estudo apresenta a incorporação das condições culturais locais em incubadoras de empresas. O ambiente de inovação tecnológica demanda sistemas de controle alinhados com processos típicos de apoio às startups, como as interações que mantém com parceiros públicos e empreendedores-clientes. Incubadoras mais permeáveis ao ambiente externo podem desenvolver controles mais competitivos.

Copyright (C) 2018 FEA-RP/USP. Todos os direitos reservados

\section{INTRODUÇÃO}

A necessidade de considerar aspectos culturais na concepção dos Sistemas de Controle Gerencial é amparada nas pesquisas de Henri (2006b), Heinicke, Guenther e Widener (2016) e Curtis e Sweeney (2017). O desafio em estudos organizacionais é conceituar cultura, dado suas naturezas distintas. Para guiar este estudo, define-se aqui 'condições culturais locais' ou apenas 'condições culturais' como a reunião de uma carga de valores históricos (advindos de fatores como raça, religião, etnia e deslocamento populacional), e de interações do ambiente no qual a incubadora se localiza, como preferências das empresas incubadas, além da própria capacidade de mudar valores à medida que diversos eventos afetam a população envolvida. Tais elementos representam diversas formas pelas quais as condições culturais afetam uma organização (Hall, 2004; Badri, Davis \& Davis, 2000).

Autor Correspondente: Tel. (48) 3721-3891

E-mail: iagofrancalopes@gmail.com (I. F. Lopes); ilse.beuren@gmail.com (I. M. Beuren)

Universidade Federal de Santa Catarina. Campus Reitor João David Ferreira Lima, s/n - Trindade, Florianópolis - SC, 88040-900, Brasil 
O relacionamento entre cultura e organização é uma via de mão dupla simultânea (Hall, 2004). Ao mesmo tempo em que a organização desenvolve internamente seus valores e práticas formando uma cultura, os indivíduos, ao interagirem com esta organização, modificam e são modificados por sua cultura. Tais indivíduos carregam consigo valores, crenças e pressupostos, ritos, rituais e cerimônias, estórias e mitos, tabus, normas e processos de comunicação, que lhes foram transmitidos ao longo do tempo e do espaço (Freitas, 1991). Havendo alguma capilaridade entre organização e ambiente, as condições culturais locais serão incorporadas à organização e aos seus sistemas.

A organização é um conjunto de regras e valores que condicionam e se manifestam em comportamentos individuais (Ribeiro \& Scapens, 2006). Para os autores, estes valores tendem a prevalecer em uma determinada configuração social, ou seja, influenciado e integrado ao ambiente em que opera. Compreender as interações com o ambiente pode contribuir para o aperfeiçoamento das estratégias e dos sistemas de gestão das organizações, e aprimorar o alinhamento dessas estratégias e sistemas às necessidades das relações neste ambiente (Lowe, 2001; Anzilago, Beuren \& Dal Vesco, 2015). Dessa forma, considera-se aqui a cultura como um fator contingencial que é capaz de moldar os Sistemas de Controle Gerencial.

Especificamente no caso observado, as incubadoras de empresas ao moldarem seus sistemas gerenciais poderiam contar com estudos que tratem a questão da influência de condições culturais locais. Tais organizações atuam no desenvolvimento de startups, especialmente negócios recém criados, que neste estágio inicial de consolidação do modelo de negócios e financeira não competem com empresas consolidadas no mercado (Leblebici \& Shah, 2004).

O presente estudo se propõe a discutir os reflexos das condições culturais locais nas alavancas de controle em incubadoras de empresas. Espera-se que os efeitos da cultura impactem de forma distinta as incubadoras, em comparação com outras organizações anteriormente estudadas. Incubadoras operam com a inovação e suporte ao desenvolvimento de modelos de negócios, reunindo operações menos rotinizadas, cuja influência das condições culturais das interações com uma continua diversidade de indivíduos envolvidos (Hall, 2004).

O estudo oferece como contribuição a análise das alavancas do Sistema de Controle Gerencial a partir de uma perspectiva abrangente, considerando as condições culturais como relevante fator contingencial (Heinicke, Guenther, \& Widener, 2016). Ainda, traz o caso das incubadoras de empresas como uma variação do modelo de atividade de suporte às startups nelas abrigadas. $\mathrm{O}$ ambiente organizacional das incubadoras se beneficiaria ao balancear a necessidade de controlar as operações das incubadas e propiciar um ambiente flexível para o desenvolvimento da pesquisa e inovação tecnológica. Assim, os resultados da pesquisa podem colaborar para a moldabilidade exigida pelos novos modelos de negócios desenvolvidos, principalmente nas políticas governamentais para o desenvolvimento da pesquisa, desenvolvimento e inovação, como é o caso do Plano Brasil Maior 2011-2014 (Lopes \& Beuren, 2016).

\section{DESENVOLVIMENTO DAS HIPÓTESES}

O Sistema de Controle Gerencial pode ser visto em diferentes perspectivas. Enquanto, Anthony (1965) atribuiu ao Sistema de Controle Gerencial atributos-chave associados ao nível hierárquico (estratégico ou organizacional, tático e operacional), Simons (1995) traz o conceito de alavancas de controle. Em Simons (1995) o Sistema de Controle Gerencial deveria compreender quatro alavancas de controle, as Levers of Control: Sistemas de Crenças; Sistemas de Restrições; Sistemas de Controle Diagnóstico; e Sistemas de Controle Interativo. Outras visões são apresentadas por Otley (1999), Malmi e Brown (2008) e Ferreira e Otley (2009).

Neste estudo, optou-se por analisar o Sistema de Controle Gerencial a partir das quatro alavancas do modelo de Simons (1995), dadas as contribuições empíricas que reconhecem a influência das contingências na adequação e uso das alavancas de controle, como de Chenhall (2003) e Heinicke, Guenther e Widener (2016).

Para Simons (1995), o Sistema de Crenças é responsável por comunicar os princípios da cultura corporativa para os funcionários da empresa. O Sistema de Restrições fornece os padrões mínimos dos relacionamentos e de execução das tarefas; a ideia básica é definir e comunicar os limites desejados de conduta e de comportamento organizacional. O Sistema de Controle Diagnóstico permite monitoramento de desempenho e, em geral, baseiase em dados quantitativos, análises estatísticas e análises da variância das metas e dos planos previamente estabelecidos. O Sistema de Controle Interativo trata de aspectos críticos no desenvolvimento das atividades operacionais, e requer comunicação face a face entre subordinados e pares (Henri, 2006a). 
O aspecto 'cultura' tornou-se importante no desenho do Sistema de Controle Gerencial, visto que muitas empresas desenvolveram operações multinacionais. Para Chenhall (2003), a cultura entra na pesquisa baseada em contingências como uma extensão dos fatores que afetam o desempenho. Há uma infinidade de significados para cultura, mas Kaplan (1965) afirma que há consenso entre os antropólogos de que a cultura é composta de tradições padronizadas e inter-relacionadas, transmitidas ao longo do tempo e do espaço por meio de mecanismos não biológicos baseados nas capacidades simbolizadoras linguísticas e não linguísticas desenvolvidas exclusivamente pelo homem.

A pesquisa em questão trata da associação da cultura como fator contingencial (Chenhall, 2003), afetando o uso das quatro alavancas dos Sistemas de Controle nas incubadoras. Primeiro, a cultura está diretamente associada com a ênfase no controle de crenças (Heinicke, Guenther e Widener, 2016; Abernethy \& Lillis, 1995; Bhimani, 2003; Henri 2006b). Embora o controle de crenças seja relevante para as organizações em geral, a flexibilidade demandada pelo ambiente inovador das incubadoras poderia afetar e ser afetado pelos controles de crenças. Neste cenário as condições culturais locais tendem a receber maior atenção dos gestores visando incentivar a inovação em modelos negócios pela comunicação formal de valores fundamentais. empresas.

$\mathbf{H}_{1}$ : A incorporação de condições culturais locais intensifica o controle de crenças nas incubadoras de

Presume-se que a diversidade de stakeholders das incubadoras de empresas, voltadas ao atendimento das demandas de desenvolvimento de pesquisa e inovação, induz os gestores a atuarem no controle de crenças, visto que o mesmo é usado para inspirar e direcionar a busca por novas oportunidades, indicar os valores que a organização pretende que sejam adotados e apontar a direção que devem seguir (Cruz, Frezatti \& Bido, 2015). A incorporação de condições culturais intensifica a declaração da missão, a comunicação dos valores fundamentais da incubadora e a ciência da força de trabalho quanto a esses elementos.

Os agentes envolvidos no ambiente das incubadoras de empresas possuem objetivos distintos. Por exemplo, os fundos de investimentos aplicam recursos para o desenvolvimento de produtos e serviços inovadores e após determinado tempo realizam o desinvestimento, portanto, o foco desses fundos é a venda de sua participação na incubada. Já a incubadora, que representa o núcleo central dessa relação em termos de desenvolvimento da empresa incubada, fornece subsídios para que a mesma, após um período de tempo, possa deixar a incubadora e se desenvolver de modo autônomo. Essa diversidade de objetivos pode ser conduzida a partir dos valores organizacionais comunicados.

Presume-se ainda haver relação entre as condições culturais e o controle de restrições. Tal presunção está consubstanciada no incentivo dado, à medida que a importância das políticas formais e rígidas na condução de processos em ambientes estáveis é reconhecida (Heinicke, Guenther \& Widener, 2016). Os controles de restrições podem tornar-se aliados benéficos, uma vez que fornecem limites à ação organizacional (Simons, 1995) na condução do relacionamento da incubadora de empresas com os demais agentes (Amato Neto, 2007).

A configuração das incubadoras de empresas demanda controles que visam limitar a ação dos agentes. Este papel de limitar e determinar as ações desejadas pela organização é exercido pelo controle de restrições (Henri, 2006a). A incorporação de condições culturais, advindas do ambiente das incubadoras, possui características de intensificar o controle de restrições, pois a liberdade concedida se restringe aos limites pré-estabelecidos (Heinicke, Guenther \& Widener, 2016).

Por outro lado, um código de conduta, que condiciona o comportamento dos agentes, é influenciado pelas condições culturais do ambiente, aspecto em geral negligenciado pela literatura de Sistemas de Controle Gerencial. Presume-se que o controle de restrições, caracterizado pela contribuição para mitigar os riscos organizacionais (Simons, 1995), é intensificado no ambiente das incubadoras à medida que ocorre a incorporação de condições culturais.

$\mathbf{H}_{2}$ : A incorporação de condições culturais locais intensifica o controle de restrições nas incubadoras de empresas.

Nas alavancas de controle de Simons (1995), o uso diagnóstico de controle é monitoramento, definido como um sistema de feedback de lógica cibernética. Neste sistema, os objetivos são definidos antecipadamente, a produção é medida, os objetivos e a saída são comparados, o feedback é fornecido, e as correções são realizadas. As incubadoras de empresas são dependentes de recursos externos, principalmente oriundos de políticas governamentais. Assim, há necessidade de diagnóstico para cumprir tais políticas e garantir a efetividade do processo como um todo, ou seja, gerar novos negócios e inovações (Barros, Claro \& Chaddad, 2009). Mesmo existindo flexibilidade nas incubadoras, o controle de diagnóstico contribui para a congruência de objetivos e de interesses de funcionários e incubadora (Heinicke, Guenther, \& Widener, 2016). 
$\mathbf{H}_{3}$ : A incorporação de condições culturais locais intensifica o uso diagnóstico de controle do Sistema de Controle Gerencial nas incubadoras de empresas.

Por fim, o sistema de controle interativo é um sistema formal usado pela alta direção de uma empresa para se envolver regularmente e pessoalmente no processo de tomada de decisão em todos os níveis operacionais da empresa (Simons, 2000). Por mais que as decisões sejam centralizadas, o gerenciamento das tarefas é realizado por meio de reuniões entre líderes de diversos setores, demandando integração de equipes e suas chefias (Jordão \& Souza, 2012).

Estudos similares têm sido realizados em ambientes inovadores (Davila \& Foster, 2005; Davila, Foster, \& Jia, 2015). O uso interativo do Sistema de Controle Gerencial daria à incubadora a flexibilidade necessária para o desenvolvimento da inovação tecnológica, desejável neste ambiente organizacional. A abertura à incorporação de condições culturais locais intensificaria e seria intensificada pela necessidade de flexibilidade e adequação às interações dos novos empreendimentos. Nos ambientes pouco rotinizados há probabilidade de elementos culturais influenciarem o escopo organizacional (Hall, 2004) como, por exemplo, nos ambientes necessariamente flexíveis das incubadoras.

$\mathbf{H}_{4}$ : A incorporação de condições culturais locais intensifica o uso interativo do Sistema de Controle Gerencial das incubadoras de empresas.

De modo geral, a influência esperada das condições culturais locais como condicionantes do uso interativo do Sistema Controle Gerencial reposiciona o sistema como receptor das mudanças do ambiente externo. Portanto, reconhecer a influência que as condições culturais exercem sobre as alavancas de controle propostas por Simons (1995) oferece contribuições à promoção da mudança e continuidade no ambiente das incubadoras de empresas.

\section{METODOLOGIA}

Esta pesquisa aplicou um questionário em gestores de 227 incubadoras de empresas nas regiões CentroOeste, Sudeste e Sul do Brasil associadas à Associação Nacional de Entidades Promotoras de Empreendimentos Inovadores (Anprotec, 2016). A amostra não probabilística por acessibilidade, das 88 respostas recebidas entre novembro de 2016 e janeiro de 2017, ao final foi formada por 75 respostas válidas. O gestor de cada incubadora foi identificado por contato telefônico à incubadora, quando solicitou-se o e-mail de seus gerentes, coordenadores e diretores executivos.

O instrumento de pesquisa (apresentado no material suplementar) compõe-se de três blocos. O primeiro bloco cobre os construtos de Sistema de Crenças e Sistema de Restrições, com questões baseadas no estudo de Widener (2007). O respondente foi solicitado a indicar em uma escala likert $(1=$ discordo totalmente a $7=$ concordo totalmente) o grau de concordância quanto cada assertiva contribuía efetivamente para o desenvolvimento organizacional interno e externo da incubadora. O segundo bloco cobre os Sistema de diagnóstico e o Sistema interativo, com questões baseadas no estudo de Henri (2006a). Os respondentes indicaram a intensidade do uso de tais instrumentos na incubadora (sendo 1 = não existe na incubadora a 7 = muito usado de modo geral).

O terceiro bloco trata das Condições Culturais que cercam a incubadora. Em Hall (2004), o ambiente no qual a organização está inserida é captado por características como aspectos históricos de raça, religião, etnia e deslocamento populacional, que afetariam a construção da identidade empresarial.

As preferências do público alvo também podem influenciar as inovações preconizadas pelas organizações (Badri, Davis \& Davis, 2000), portanto, neste bloco são consideradas as variações de preferência dos empreendedoresclientes das incubadoras. Incluem-se ainda a capacidade da organização em mudar os valores culturais e a forma com que os valores e normas se alteram à medida que os eventos afetam os stakeholders envolvidos. Para este bloco solicitou-se que o respondente indicasse o quanto cada condição cultural local é incorporada e está presente internamente na incubadora em que atua $(1=$ pouco presente e $7=$ muito presente $)$.

Os construtos foram tratados de modo unidimensional, com consistência interna validada por análise fatorial confirmatória. Para testar as hipóteses aplicou-se a técnica de Modelagem de Equações Estruturais, apropriada para compreender relações complexas (Hair Jr., Hult, Ringle \& Sarstedt, 2014). Os modelos estruturais enfatizam os construtos latentes, a natureza e a magnitude das relações entre construtos (Hair Jr., Black, Babin, Anderson \& Tatham, 2009). Os parâmetros dessas relações indicam o efeito das variáveis independentes sobre as variáveis dependentes (Marôco, 2010). 
A aplicação da Modelagem de Equações Estruturais exige um modelo de mensuração e avaliação do modelo estrutural (Ringle, Silva \& Bido, 2014). Assim, após a constatação de que as medidas do modelo de mensuração apresentam-se adequadas, deve-se avaliar o indicador de ajuste geral do modelo, a saber: (i) Coeficiente de Determinação de Pearson $\left(\mathrm{R}^{2}\right)$; (ii) Indicador de Stone-Geisser ou Relevância Preditiva; e (iii) Índice de Adequação do Modelo (Goodness of Fit).

A amostra do estudo foi estimada por meio do G*Power 3.1.9 (Faul, Erdfelder, Buchner, \& Lang, 2009). Considerando o construto que recebe o maior número de relações (setas), que nesta situação é 1 , com um $\alpha=0,05$ de significância, com poder explicativo de $0,80, \mathrm{f}^{2}$ mediano $=0,15$, a amostra atende ao número mínimo de 55 respondentes e permite a análise pelo Partial Least Squares via bootstrapping.

Na modelagem não se assume que os dados estejam normalmente distribuídos. Desse modo, o processo de bootstrapping ou reamostragem é desenhado aleatoriamente (com substituição) do conjunto de dados original (Hair Jr. et al., 2014). Cada sequência é usada para estimar os coeficientes de caminhos para as relações diretas e indiretas, além de fornecer maior confiabilidade aos resultados. Esse processo é repetido até que um número de subamostras seja criado, no caso foi repetido 500 vezes, com reposição. Como parâmetros para explicar o teste, Cohen (1988) menciona que, em pesquisas das Ciências Sociais, o $\mathrm{R}^{2}$ de $2 \%$ representa um pequeno efeito, 13\% um efeito médio, e $26 \%$ um efeito grande.

\subsection{Descrição da amostra}

A Tabela 1 apresenta o perfil dos respondentes do questionário. São 75 empregados de 75 incubadoras em sua maioria de pequeno porte.

Tabela 1. Perfil dos respondentes e das incubadoras pesquisadas

\begin{tabular}{|c|c|c|c|c|c|}
\hline Gênero & Quant. & $\%$ & Idade & Quant. & $\%$ \\
\hline Feminino & 36 & 48,00 & Até 31 anos & 18 & 24,00 \\
\hline Masculino & 39 & 52,00 & De 32 a 38 anos & 20 & 26,67 \\
\hline Formação & Quant. & $\%$ & De 39 a 47 anos & 16 & 21,33 \\
\hline Graduação & 29 & 38,67 & De 48 a 68 anos & 18 & 24,00 \\
\hline MBA/Especialização & 5 & 6,67 & Não responderam & 03 & 4,00 \\
\hline Mestrado & 6 & 8,00 & Cargo & Quant. & $\%$ \\
\hline Doutorado & 3 & 4,00 & Assistente & 15 & 20,00 \\
\hline Pós-doutorado & 1 & 1,33 & Analista & 8 & 10,67 \\
\hline Não responderam & 31 & 41,33 & Gerente & 23 & 30,67 \\
\hline \multirow{2}{*}{ Tempo no cargo } & \multirow{2}{*}{ Quant. } & \multirow{2}{*}{$\%$} & Coordenador & 21 & 28,00 \\
\hline & & & Diretor Executivo & 8 & 10,67 \\
\hline Até 11 meses & 8 & 10,67 & Porte das incubadoras & Quant. & $\%$ \\
\hline De 1 a 5 anos & 46 & 61,33 & De 1 a 5 empregados & 52 & 69,33 \\
\hline De 6 a 10 anos & 12 & 16,00 & De 6 a 10 empregados & 17 & 22,67 \\
\hline De 11 a 15 anos & 6 & 8,00 & De 11 a 15 empregados & 3 & 4,00 \\
\hline De 16 a 20 anos & 2 & 2,67 & De 16 ou mais empregados & 3 & 4,00 \\
\hline
\end{tabular}

Fonte: Elaboração própria.

Os respondentes participantes da pesquisa se distribuem nas faixas de 31 a 68 anos de idade, e nos gêneros. Mas nota-se uma concentração quanto à formação e tempo no cargo. Cerca de $41 \%$ deles não preencheram a formação acadêmica, e 38,67\% não avançaram os estudos além da graduação. Ainda, 70\% deles não têm mais de 5 anos de tempo no cargo. Quanto ao porte das incubadoras, quase $70 \%$ são de pequeno porte, reunindo até 5 empregados.

A Tabela 2 a seguir apresenta as médias obtidas em cada uma das questões., apresentam-se as estatísticas descritivas das questões. 
Tabela 2. Estatística descritiva das questões da pesquisa

\begin{tabular}{|c|c|c|c|c|c|}
\hline Questões & Mínimo & Máximo & Média & D. Padrão & D. padrão/média \\
\hline \multicolumn{6}{|l|}{ Sistema de Crenças } \\
\hline Clareza de comunicação da missão da incubadora & 1 & 7 & 5,49 & 1,37 & 0.25 \\
\hline Clareza de comunicação dos valores da incubadora pelos gestores & 1 & 7 & 5,55 & 1,33 & 0.24 \\
\hline Empregados cientes dos valores da incubadora & 2 & 7 & 5,74 & 1,23 & 0.21 \\
\hline A declaração de missão da incubadora inspira a força de trabalho & 1 & 7 & 5,47 & 1,44 & 0.26 \\
\hline \multicolumn{6}{|l|}{ Sistemas de Restrições } \\
\hline A incubadora utiliza código de conduta & 1 & 7 & 5,23 & 1,57 & 0.30 \\
\hline O código de conduta informa comportamentos indesejáveis & 1 & 7 & 5,26 & 1,51 & 0.29 \\
\hline Riscos a serem evitados são informados & 1 & 7 & 4,92 & 1,59 & 0.32 \\
\hline Empregados estão ciente do código de conduta & 1 & 7 & 5,06 & 1,61 & 0.32 \\
\hline \multicolumn{6}{|l|}{ Sistemas de Controle Diagnóstico (intensidade de uso para) } \\
\hline Acompanhar o progresso das atividades em direção às metas & 1 & 7 & 5,30 & 1,62 & 0.31 \\
\hline Monitorar os resultados & 1 & 7 & 5,51 & 1,35 & 0.25 \\
\hline Comparar os resultados com as expectativas & 1 & 7 & 5,09 & 1,54 & 0.30 \\
\hline Revisar as medidas chave de desempenho & 1 & 7 & 5,13 & 1,53 & 0.30 \\
\hline \multicolumn{6}{|l|}{ Sistema de Controle Interativo (intensidade de uso para) } \\
\hline Promover a discussão entre superiores, subordinados e pares & 1 & 7 & 5,40 & 1,29 & 0.24 \\
\hline Discutir dados subjacentes, pressupostos e os planos de ação & 1 & 7 & 5,12 & 1,40 & 0.27 \\
\hline Fornecer uma visão comum da incubadora & 2 & 7 & 5,55 & 1,27 & 0.23 \\
\hline Oportunizar a incubadora concentrar-se em questões comuns & 1 & 7 & 5,42 & 1,24 & 0.23 \\
\hline Possibilitar o foco em fatores críticos de sucesso & 1 & 7 & 5,32 & 1,41 & 0.27 \\
\hline Desenvolver um vocabulário comum na incubadora & 1 & 7 & 5,45 & 1,33 & 0.24 \\
\hline \multicolumn{6}{|l|}{ Incorporação de Condições Culturais locais } \\
\hline Valores históricos (raça, religião, etnia e deslocamento populacional) & 1 & 7 & 3,82 & 1,78 & 0.47 \\
\hline Ambiente no qual a incubadora se localiza & 2 & 7 & 5,81 & 1,15 & 0.20 \\
\hline Variação do gosto e preferências dos clientes & 1 & 7 & 4,88 & 1,38 & 0.28 \\
\hline Organizações com capacidade de mudar os valores culturais & 1 & 7 & 4,74 & 1,53 & 0.32 \\
\hline Valores e normas que se alteram com fatos externos e eventos & 1 & 7 & 4,74 & 1,50 & 0.32 \\
\hline
\end{tabular}




\section{ANÁLISE E DISCUSSÃo DOS RESULTADOS}

\subsection{Análises iniciais}

A Tabela 3 apresenta como os construtos se comportam em intervalos. O primeiro intervalo representa aquele com a menor nota atribuída a cada uma das quatro alavancas de controle do SCG, e o último intervalo aquele com a maior nota atribuída pelos respondentes. A segregação foi realizada pela soma da pontuação dentro de cada construto do Sistema de Controle Gerencial e da incorporação das condições culturais locais. Como exemplo, se o construto de Sistema de Crenças possui quatro assertivas (em escala de 1 a 7 ) o somatório de um respondente varia de 4 a 28. Configura-se neste casos os intervalos de 4-10; 10-16; 16-22 e 22-28.

Tabela 3. Estatística descritiva dos construtos segregada em intervalos

\begin{tabular}{|c|c|c|c|c|c|c|}
\hline Construtos & $\begin{array}{l}\text { Primeiro e ultimo de } \\
\text { somatória dos itens }\end{array}$ & $\begin{array}{c}\text { Quantidade de } \\
\text { incubadoras } \\
\text { no intervalo }\end{array}$ & $\begin{array}{l}\text { Soma } \\
\text { mínima }\end{array}$ & $\begin{array}{l}\text { Soma } \\
\text { máxima }\end{array}$ & $\begin{array}{l}\text { Média do } \\
\text { construto }\end{array}$ & $\begin{array}{l}\text { Desvio } \\
\text { Padrão }\end{array}$ \\
\hline \multirow{2}{*}{ Sistema de Crenças } & $1^{\circ}$ Intervalo $4-10$ & 1 & 1 & 4 & 2,80 & 2,68 \\
\hline & $4^{\circ}$ Intervalo $22-28$ & 47 & 4 & 7 & 6,34 & 0,71 \\
\hline \multirow{2}{*}{ Sistema de Restrições } & $1^{\circ}$ Intervalo $4-10$ & 5 & 1 & 6 & 2,05 & 1,15 \\
\hline & $4^{\circ}$ Intervalo $22-28$ & 35 & 4 & 7 & 6,39 & 0,65 \\
\hline \multirow{2}{*}{$\begin{array}{l}\text { Sistema de Controle } \\
\text { Diagnóstico }\end{array}$} & $1^{\circ}$ Intervalo $4-10$ & 4 & 1 & 7 & 2,38 & 1,93 \\
\hline & $4^{\circ}$ Intervalo $22-28$ & 40 & 4 & 7 & 6,31 & 0,70 \\
\hline \multirow{2}{*}{$\begin{array}{l}\text { Sistema de Controle } \\
\text { Interativo }\end{array}$} & $1^{\circ}$ Intervalo $6-15$ & 1 & 1 & 7 & 3,43 & 4,39 \\
\hline & $4^{\circ}$ Intervalo $34-42$ & 31 & 3 & 7 & 6,40 & 0,68 \\
\hline \multirow{2}{*}{$\begin{array}{l}\text { Incorporação das } \\
\text { Condições Culturais }\end{array}$} & $1^{\circ}$ Intervalo $5-12$ & 1 & 1 & 4 & 2,00 & 1,22 \\
\hline & $4^{\circ}$ Intervalo $27-35$ & 25 & 2 & 7 & 6,03 & 1,00 \\
\hline
\end{tabular}

Na segregação em intervalos, o maior grupo de incubadoras está no $4^{\circ}$ intervalo (22 a 28). O Sistema de Crenças mostra-se relevante e tem forte concordância quanto a tal relevância entre os respondentes (Média= 6,34; Desvio Padrão= 0,71). Sobretudo em relação à declaração de missão da incubadora comunicar claramente os valores fundamentais da mesma. Sugerindo uma ação por parte dos gestores de comunicar os valores fundamentais da incubadora e seu conhecimento pela força de trabalho. Além disso, há concordância quanto à missão inspirar a força de trabalho da incubadora.

No construto Sistema de Restrições (Média=6,39 Desvio Padrão=0,65) é perceptível forte concordância quanto à incubadora se basear em um código de conduta, para definir o comportamento apropriado de sua força de trabalho, e informar os comportamentos que estão fora dos limites apregoados pela incubadora. Há, ainda, concordância quanto à presença de um sistema que comunica à força de trabalho os riscos que devem ser evitados. Por fim, concorda-se quanto à ciência da força de trabalho sobre a existência de um código de conduta da incubadora.

O Sistema de Controle Diagnóstico apresenta elevado uso em âmbito interno e externo da incubadora (Média=6,31; Desvio Padrão=0,70). Acompanhar o progresso das atividades em direção às metas, monitorar os resultados, comparar os resultados com as expectativas e revisar as medidas chave de desempenho são atividades relevantes para o suporte das incubadoras. Como as incubadoras de empresas são dependentes de recursos externos para o seu funcionamento, principalmente, advindos de fontes públicas, como da Financiadora de Estudos e Projetos (FINEP), há necessidade de controles para viabilizar suas operações (Jordão \& Souza, 2012). Desse modo, controles mais rígidos, como os do Sistema de Controle Diagnóstico, são indicados para este fim.

O Sistema de Controle Interativo também apresenta elevado uso pelos gestores das incubadoras de empresas (Média=6,60; Desvio Padrão=0,68). Promover a discussão em reuniões com superiores, subordinados e pares, proporcionar o contínuo desafio e discutir os planos de ação, fornecer uma visão comum da incubadora, oportunizar à incubadora concentrar-se em questões comuns, possibilitar à organização focalizar-se nos fatores críticos de sucesso e desenvolver um vocabulário comum na incubadora são ações praticadas no âmbito interno e externo da incubadora. 
Contudo, ao observar o grau de incorporação das Condições Culturais locais à incubadora, apesar da forte presença geral (Média=6,03; Desvio Padrão= 1,36), percebe-se certa ambiguidade entre os respondentes quanto à influência de valores históricos. Uma maior concordância é observada em relação à influência do ambiente onde a incubadora se localiza. Ainda há influência da variação das preferências dos clientes das incubadoras, da capacidade em mudar os valores culturais frente a eventos externos.

Na Figura 1, apresenta-se um boxplot da relação dos construtos da pesquisa, cada uma das alavancas de controle do Sistema de Controle Gerencial com a incorporação das Condições Culturais locais.

Interessante notar nos diagramas boxplot da Figura 1, que a tendência "quanto maior a incorporação de condições culturais" maior a adoção, uso e força das alavancas de controle, é mais evidente para os sistemas de crenças, restrições e diagnóstico. No caso dos sistemas de restrições e de diagnóstico, existe a tendência que para baixa influência cultural, haverá baixo uso desses sistemas.

Já para o sistema de crenças, um acréscimo na influência cultural eleva a clareza e a disseminação deste sistema, mas o efeito moderador é mais modesto se comparado aos de diagnóstico e de restrições. O interativo apresenta um maior nível de uso, mesmo em incubadoras em que denotam baixa permeabilidade em relação ao ambiente local. Ainda, para os sistemas de restrição, diagnóstico e interativo, altos graus de interação com fatores culturais apresentam ambiguidade na adoção e uso de tais sistemas, o que não acontece para o sistema de crenças. 
Figura 1. Boxplot dos construtos da pesquisa

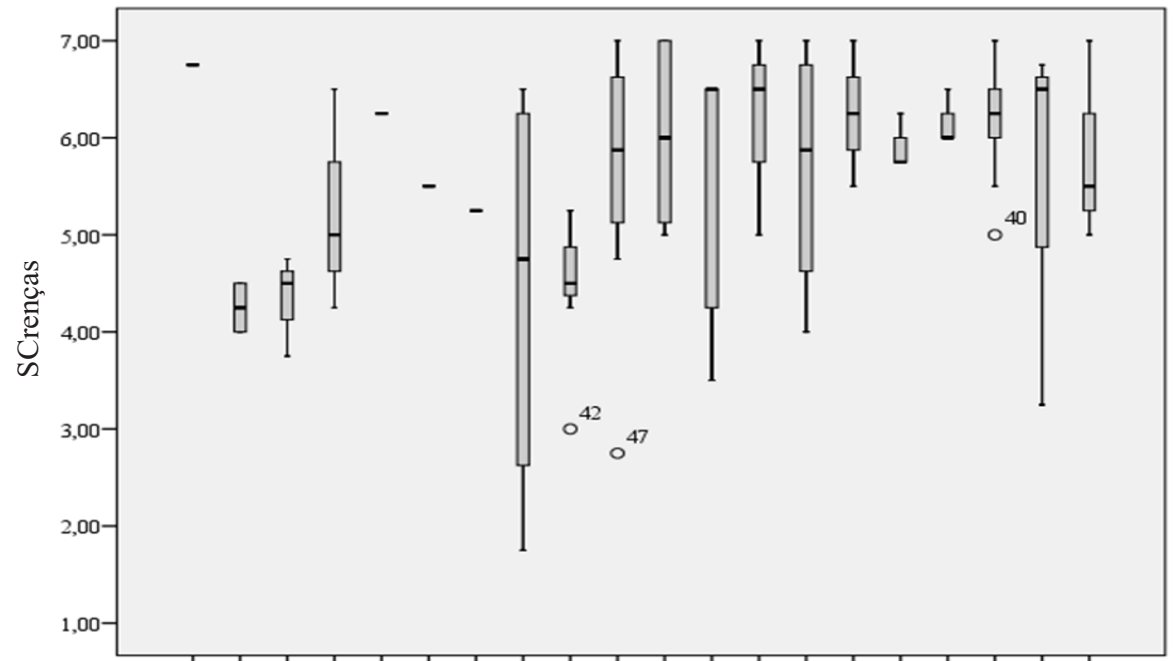

2,00 2,60 2,80 3,20 3,40 3,60 3,80 4,00 4,20 4,40 4,60 4,80 5,00 5,20 5,40 5,60 5,80 6,00 6,60 7,00 CCulturais

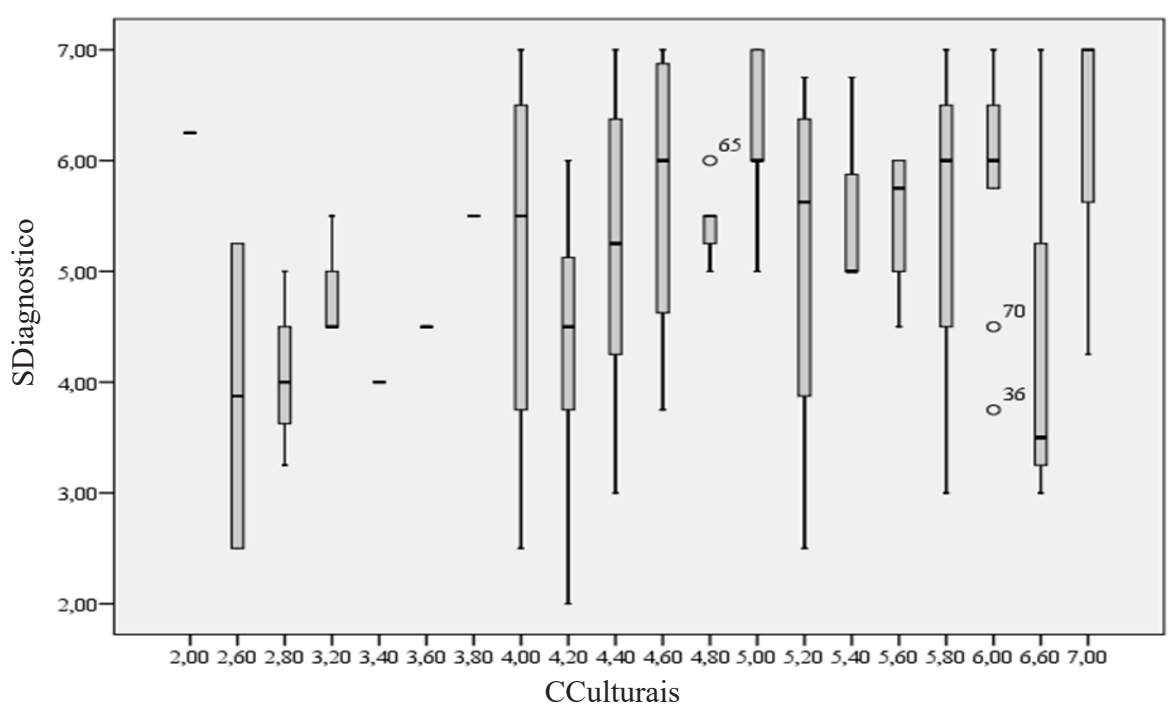

Fonte: Elaboração própria.

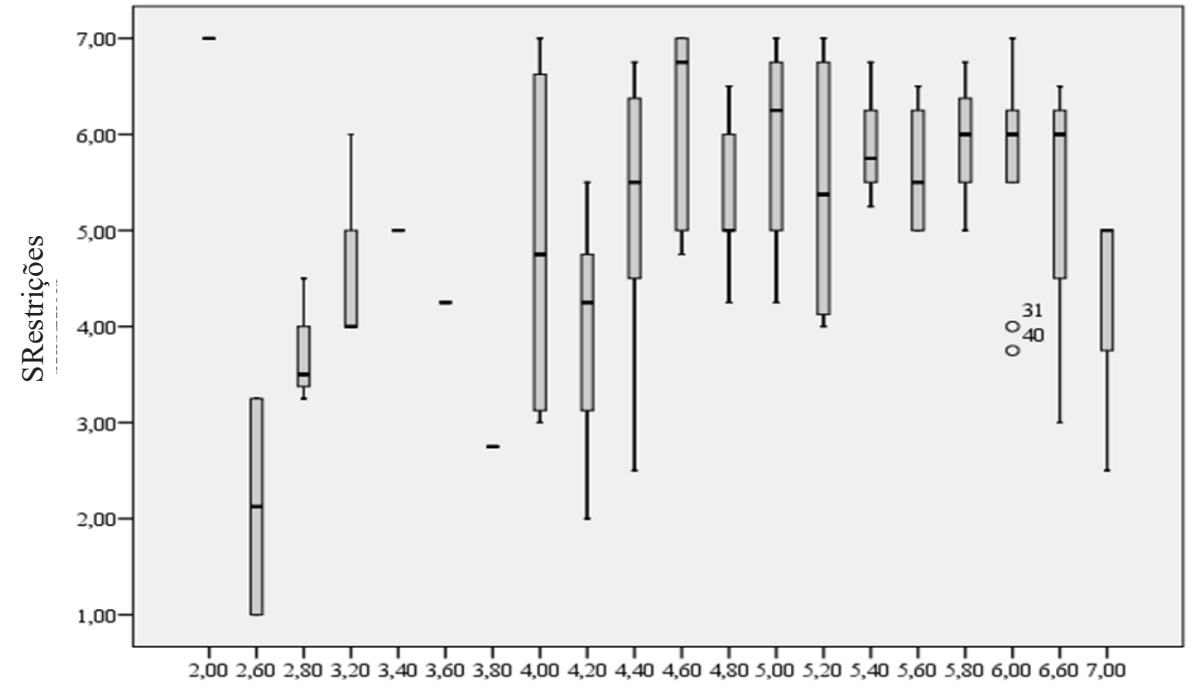

CCulturais

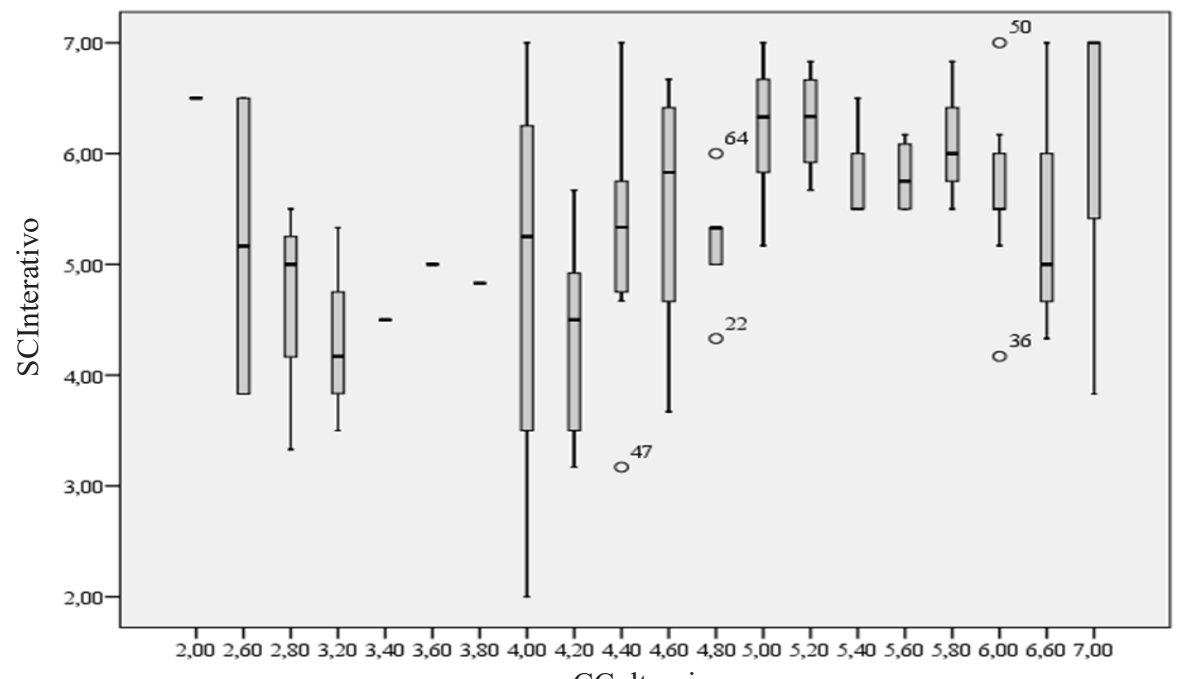

CCulturais 


\subsection{Teste das Hipóteses de Pesquisa}

Na Tabela 4 apresentam-se os resultados da Modelagem de Equações Estruturais. Dado que o teste de GoF não é adequado para investigações que utilizam a modelagem de equações estruturais (Hair Jr. et al., 2014), empregou-se o Stone-Geisser ou Relevância Preditiva e Coeficiente de Determinação de Pearson $\left(\mathrm{R}^{2}\right)$. Adicionalmente, verificou-se os coeficientes de caminhos e significância dos reflexos das condições culturais nas alavancas de controle (Simons, 1995), pelo bootstrapping.

Tabela 4. Modelo estrutural - Alavancas de controle e Condições culturais

\begin{tabular}{|c|c|c|c|c|}
\hline Relação estrutural & $\begin{array}{c}\text { Coeficiente } \\
\text { estrutural }\end{array}$ & $\begin{array}{c}\text { Erro } \\
\text { padrão }\end{array}$ & Estatística T & P Value \\
\hline Condições Culturais $\rightarrow$ Sistema de Crenças & 0,313 & 0,093 & 3,375 & $\mathbf{0 , 0 0 1 *}$ \\
\hline Condições Culturais $\rightarrow$ Sistemas de Restrições & 0,272 & 0,128 & 2,126 & $0,034 * *$ \\
\hline Condições Culturais $\rightarrow$ Sistema Diagnóstico de Controle & 0,259 & 0,105 & 2,462 & $0,014 * *$ \\
\hline Condições Culturais $\rightarrow$ Sistema Interativo de Controle & 0,338 & 0,093 & 3,645 & $0,000 *$ \\
\hline $\mathrm{R}^{2}$ Sistema de Crenças & & & & 0,098 \\
\hline $\mathrm{R}^{2}$ Sistema de Restrições & & & & 0,074 \\
\hline $\mathrm{R}^{2}$ Sistema de Controle Diagnóstico & & & & 0,067 \\
\hline $\mathrm{R}^{2}$ Sistema de Controle Interativo & & & & 0,114 \\
\hline Relevância Preditiva - Sistema de Crenças & & & & 0,068 \\
\hline Relevância Preditiva - Sistema de Restrições & & & & 0,049 \\
\hline Relevância Preditiva - Sistema de Controle Diagnóstico & & & & 0,035 \\
\hline Relevância Preditiva - Sistema de Controle Interativo & & & & 0,065 \\
\hline
\end{tabular}

Fonte: Elaboração própria.

Nota: *Significante a $1 \% ; *$ Significante a $5 \%$.

De forma geral, o teste nos permite aceitar todas as hipóteses. Contudo, é necessária uma breve análise dos limites desses resultados. A incorporação de condições culturais do ambiente local está positivamente associada com a clareza e entendimento da declaração de visão e missão, o que mostraria um maior efeito do controle de crenças $\left(\mathrm{H}_{1}\right.$ aceita). Tal resultado é similar ao encontrado em Heinicke, Guenther e Widener (2016) para empresas alemãs.

O sistema de crenças demanda interação constante com o ambiente local. Pois, à medida que os eventos no ambiente da incubadora de empresas ocorrem e envolvem agentes a ela relacionados, há necessidade inclusive de acompanhamento das preferências dos stakeholders em relação aos valores organizacionais. Os gestores levariam em consideração estes valores para usar e adequar o Sistema de Controle Gerencial, assim como os processos organizacionais (Henri, 2006b).

O código de conduta, como instrumento de controle de restrições também apresenta associação positiva com a incorporação dos aspectos culturais locais $\left(\mathrm{H}_{2}\right.$ aceita). Esse resultado pode ser explicado pela influência das condições culturais locais sobre o sistema de restrição, pois a incubadora interage com empreendedores, os quais possuem diferentes visões sobre como desenvolver novos produtos e negócios, que por vezes não se alinham com a conduta e limitações preconizados pela incubadora de empresa. Assim, tais restrições de comportamento direcionam o relacionamento entre incubadora e incubadas.

Nesse cenário, o sistema de restrições é um atributo organizacional desenhado e redesenhado pelo ambiente, para que o uso seja intensificado internamente. O controle de diagnóstico pretende monitorar e comparar as expectativas com os resultados realizados, privilegiando a revisão das medidas chaves de desempenho (Simons, 1995). A incubadora é produto de múltiplas interações com os agentes organizacionais, como universidades e órgãos de fomento (Lopes, 2017), que possuem objetivos distintos, em um ambiente onde as condições culturais não são constantes (Hall, 2004).

Em um ambiente de rápidas mudanças de inovação e tecnologia, funcionários teriam de acompanhar metas, monitorar resultados, comparar resultados com as expectativas e revisar medidas de desempenho para manter a competitividade. Tal competitividade, no caso de prestadores desse tipo de serviço, demanda atender expectativas de clientes e de parceiros de negócios. 
O que naturalmente faz com que a integração de condições culturais locais, presentes nos relacionamentos que mantém com parceiros e clientes, esteja positivamente associada com o sistema de diagnóstico ( $\mathrm{H}_{3}$ aceita). Já em relação ao sistema interativo, um interessante resultado pode ser destacado. A maior interação entre supervisores e empregados, levando a uma visão e vocabulário comuns e constante debate dos rumos a serem tomados, é algo que está mais associado ao ambiente interno da incubadora de empresa. O resultado estatístico do teste nos leva a aceitar uma associação positiva entre o desenvolvimento desse ambiente mais interativo e a incorporação de cultura local, vinda da interação com stakeholders da incubadora (aceitar $\mathrm{H}_{4}$ ). Contudo, destaca-se como observado na Figura 1 que o sistema interativo apresenta um maior nível de uso mesmo em incubadoras em que denotam baixa permeabilidade em relação ao ambiente local.

O sistema interativo é desenvolvido mesmo nestas condições de baixa permeabilidade. Atributos de cultura organizacional, associados ao tipo de organização em questão, como inovação, orientação para resultados e controle rígido versus controle flexível, explicam a adoção das práticas gerenciais (Baird, Harrison \& Reeve, 2004). Assim, o desenvolvimento do controle interativo poderia decorrer da própria atividade desenvolvida pela incubadora.

No caso em questão, incubadoras possuem características específicas do setor em que atuam, sendo demandadas por ofertar soluções sob medida para as empresas sob sua tutela, lidar com diferentes modelos de negócios, criando um ambiente de inovação e flexibilidade. Tais características internas associadas ao próprio negócio de apoio ao desenvolvimento de startups demandas características especificas das alavancas de controle (Baird, Harrison \& Reeve, 2004).

\section{CONCLUSÕES}

Os resultados da pesquisa revelam que a incorporação de condições culturais intensifica o uso das alavancas de controle propostas por Simons (1995). As condições culturais locais das organizações deveriam ser consideradas como fatores contingenciais para uma melhor compreensão de como as organizações, que atuam em ambiente de desenvolvimento e tecnologia, como as incubadoras de empresas, gerenciam, controlam e criam um ambiente flexível para inovação tecnológica.

O estudo apresenta uma proposta de mensuração das condições culturais locais dessas organizações. O conceito foi composto questionando aos empregados o quanto a organização incorporaria e seria influenciada por valores históricos, como raça, religião, etnia e deslocamento populacional. Além disso, foi necessário considerar o ambiente em que a incubadora se localiza, as preferências dos clientes (empresas incubadas e seus empreendedores), e a capacidade de mudança de valores dado eventos externos.

Os resultados mostram que a incorporação de tais condições está positivamente associada com a extensão da influência das alavancas de controle, ou com seu uso. A clareza da missão e visão aos empregados no sistema de crenças, o conhecimento das restrições dos códigos de conduta, o uso e revisão de métricas de desempenho pelas equipes, e a maior interação das equipes na discussão de estratégias e soluções, são mais presentes em incubadoras mais propensas a estarem abertas ao ambiente externo. O que mostra certa permeabilidade ao ambiente, destacando o efeito da cultural local como fator contingencial relevante.

O estudo também fornece implicações práticas. Os gestores das incubadoras de empresas devem se atentar às condições culturais presentes no ambiente. Por exemplo, pode ser mais fácil criar um ambiente flexível, propício para a pesquisa, desenvolvimento e inovação, ao considerarem variações de gostos e preferências das empresas incubadas (Badri, Davis \& Davis 2000). Assim, antes de realizar a intensificação do uso das alavancas de controle, encoraja-se as incubadoras de empresas a rever seu grau de permeabilidade ao ambiente, de forma a considerar cada vez mais as contingências ali presentes.

\section{REFERÊNCIAS}

Abernethy, M. A., \& Lillis, A. M. (1995). The impact of manufacturing flexibility on management control system design. Accounting, Organizations and Society, 20(4), 241-258. DOI: https://doi.org/10.1016/0361-3682(94) E0014-L.

Amato Neto, J. (2007). As políticas industriais e tecnológicas e as pequenas e médias empresas: iniciativas na história recente da economia brasileira. Revista Gestão Industrial, 3(1), 87-102. DOI: 10.3895/S180804482007000100008 . 
Anthony, R. N. (1995). Management planning and control systems: A framework for analysis. Boston: Harvard Business School Press.

Anzilago, M., Beuren, I. M., \& Dal Vesco. (2015). O uso dos sistemas de controle gerencial nas medidas de desempenho está associada com a diminuição da ambiguidade de papéis e aumento de empowerment psicológico? Anais do Congresso ANPCONT, Curitiba, PR, Brasil, 9.

Associação Nacional de Entidade Promotoras de Empreendimentos Inovadores (ANPROTEC). (2016). Estudo de impacto econômico: segmento de incubadoras de empresas do Brasil. Brasília: ANPROTEC: SEBRAE. Recuperado em 12 janeiro, 2017, de http://www.anprotec.org.br/Relata/18072016\%20Estudo_ANPROTEC_ v6.pdf

Badri, M. A., Davis, D., \& Davis, D. (2000). Operations strategy, environmental uncertainty and performance: a path analytic model of industries in developing countries. Omega, 28(2), 155-173. DOI: https://doi. org/10.1016/S0305-0483(99)00041-9.

Baird, K. M., Harrison, G. L., \& Reeve, R. C. (2004). Adoption of activity management practices: a note on the extent of adoption and the influence of organizational and cultural factors. Management Accounting Research, 15(4), 383-399. DOI: https://doi.org/10.1016/j.mar.2004.07.002.

Barros, H. M., Claro, D. P., \& Chaddad, F. R. (2009). Políticas para a inovação no Brasil: efeitos sobre os setores de energia elétrica e de bens de informática. Revista de Administração Pública, 43(6), 1459-1486.

Bhimani, A. (2003). A study of the emergence of management accounting system ethos and its influence on perceived system success. Accounting, Organizations and Society, 28(6), 523-548. DOI: https://doi. org/10.1016/S0361-3682(02)00025-9.

Chenhall, R. H. (2003). Management control systems design within its organizational context: findings from contingency-based research and directions for the future. Accounting, Organizations and Society, 28(2), 127168. DOI: https://doi.org/10.1016/S0361-3682(01)00027-7.

Cruz, A. P. C., Frezatti, F., \& de Souza Bido, D. (2015). Estilo de liderança, controle gerencial e inovação: Papel das alavancas de controle. RAC. Revista de Administração Contemporânea, 19(6), 772-794. DOI: http:// dx.doi.org/10.1590/1982-7849rac2015150099.

Curtis, E., \& Sweeney, B. (2017). Managing different types of innovation: mutually reinforcing management control systems and the generation of dynamic tension. Accounting and Business Research, 47(3), 313-343. DOI: https://doi.org/10.1080/00014788.2016.1255585.

Davila, A., \& Foster, G. (2005). Management accounting systems adoption decisions: evidence and performance implications from early-stage/startup companies. The Accounting Review, 80(4), 1039-1068. DOI: https:// doi.org/10.2308/accr.2005.80.4.1039.

Davila, A., Foster, G., \& Jia, N. (2015). The valuation of management control systems in start-up companies: international field-based evidence. European Accounting Review, 24(2), 207-239. DOI: https://doi.org/10.10 80/09638180.2014.965720.

Faul, F., Erdfelder, E., Buchner, A., \& Lang, A. G. (2009). Statistical power analyses using G*Power 3.1: Tests for correlation and regression analyses. Behavior Research Methods, 41(4), 1149-1160. DOI: https://doi. org/10.3758/BRM.41.4.1149.

Ferreira, A. \& Otley, D. (2006). Exploring inter and intra-relationships between the design and use of management control system. SSRN.

Ferreira, A., \& Otley, D. (2009). The design and use of performance management systems: An extended framework for analysis. Management Accounting Research, 20(4), 263-282. DOI: https://doi.org/10.1016/j. mar.2009.07.003

Fornell, C., \& Larcker, D. F. (1981). Structural equation models with unobservable variables and measurement error: Algebra and statistics. Journal of Marketing Research, 18(3). 382-388. DOI: 10.2307/3150980.

Freitas, M. E. (1991). Cultura organizacional: formação, tipologias e impacto. São Paulo: McGraw-Hill.

Hair Jr., J. F., Hult, G. T. M., Ringle, C. M. \& Sarstedt, M. (2014). A primer on partial least squares structural equation modeling (PLS-SEM). Thousand Oaks: Sage. 
Hair Jr, J. F., Black, W. C., Babin, B. J., Anderson, R. E., \& Tatham, R. L. (2009). Análise multivariada de dados. São Paulo: Bookman.

Hall, R. H. (2004). Organizações: estruturas, processos e resultados. São Paulo: Pearson Prentice Hall.

Heinicke, A., Guenther, T. W., \& Widener, S. K. (2016). An examination of the relationship between the extent of a flexible culture and the levers of control system: The key role of beliefs control. Management Accounting Research, 33(s/n), 25-41. DOI: https://doi.org/10.1016/j.mar.2016.03.005.

Henri, J. F. (2006a). Management control systems and strategy: A resource-based perspective. Accounting, Organizations and Society, 31(6), 529-558. DOI: https://doi.org/10.1016/j.aos.2005.07.001.

Henri, J. F. (2006b). Organizational culture and performance measurement systems. Accounting, Organizations and Society, 31(1), 77-103. DOI: https://doi.org/10.1016/j.aos.2004.10.003.

Jordão, R. V. D., \& de Souza, A. A. (2012). Efeitos da cultura corporativa no sistema de controle gerencial pósaquisição: um estudo de sucesso numa empresa brasileira. REGE. Revista de Gestão, 19(1), 55-71. DOI: http://dx.doi.org/10.5700/issn.2177-8736.rege.2012.49848.

Kaplan, D. (1965). The superorganic: science or metaphysics. American Anthropologist, 67, 958-976. DOI: https:// doi.org/10.1525/aa.1965.67.4.02a00060.

Leblebici, H., \& Shah, N. (2004). The birth, transformation and regeneration of business incubators as new organisational forms: understanding the interplay between organisational history and organisational theory. Business History, 46(3), 353-380. DOI: https://doi.org/10.1080/0007679042000219175a.

Lopes, I. F. (2017). Influência de pressões ambientais na formação de redes mediada pelo sistema de controle gerencial em incubadoras de empresas. Dissertação de Mestrado, Universidade Federal de Santa Catarina, Florianópolis, SC, Brasil.

Lopes, I. F., \& Beuren, I. M. (2016). Evidenciação da Inovação no Relatório da Administração: uma análise na perspectiva da Lei do Bem (Lei No. 11.196/2005). Perspectivas em Gestão \& Conhecimento, 6(1), 109-127.

Lowe, A. (2001). After ANT-An illustrative discussion of the implications for qualitative accounting case research. Accounting, Auditing \& Accountability Journal, 14(3), 327-351. DOI: https://doi.org/10.1108/ EUM0000000005519.

Malmi, T., \& Brown, D. A. (2008). Management control systems as a package-Opportunities, challenges and research directions. Management Accounting Research, 19(4), 287-300. DOI: https://doi.org/10.1016/j. mar.2008.09.003.

Marôco, J. (2010). Análise de equações estruturais: fundamentos teóricos, software \& aplicações. Pêro Pinheiro: ReportNumber.

Otley, D. (1999). Performance management: a framework for management control systems research. Management Accounting Research, 10(4), 363-382. DOI: https://doi.org/10.1006/mare.1999.0115.

Ribeiro, J. A., \& Scapens, R. W. (2006). Institutional theories in management accounting change: contributions, issues and paths for development. Qualitative Research in Accounting \& Management, 3(2), 94-111. DOI: https://doi.org/10.1108/11766090610670640.

Ringle, C. M., Silva, D., \& Bido, D. D. S. (2014). Modelagem de equações estruturais com utilização do SmartPLS. REMark, 13(2), 54. DOI: 10.5585/remark.v13i2.2717.

Simons, R. (1995). Levers of control. Cambridge: Harvard Business School Publishing.

Simons, R. (2000). Performance measurement and control systems for implementing strategy. New Jersey: Prentice Hall.

Widener, S. K. (2007). An empirical analysis of the levers of control framework. Accounting, Organizations and Society, 32(7), 757-788. DOI: https://doi.org/10.1016/j.aos.2007.01.001.

\section{Como citar este artigo}

Lopes, I. F. \& Beuren, I. M. (2018). Reflexos da cultura local nos sistemas de controle de incubadoras de empresas. Revista de Contabilidade e Organizações, 12:e142273. DOI: http://dx.doi.org/10.11606/ issn.1982-6486.rco.2018.142273 


\title{
REFLEXOS DA CULTURA LOCAL NOS SISTEMAS DE CONTROLE DE INCUBADORAS DE EMPRESAS
}

\author{
MATERIAL SUPLEMENTAR
}

A seguir são apresentadas as questões do questionário, da forma como foram aplicadas, segregadas por construto. Todos os itens foram respondidos em escala likert de 7 pontos.

\section{Construtos e Questões}

Assinale seu grau de concordância quanto a contribuição de cada assertiva para o desenvolvimento organizacional interno e externo da incubadora. Atribua notas de " 1 " a "7", sendo 1 = Discordo totalmente, 7 $=$ Concordo totalmente.

\section{Sistema de Crenças}

1. Os gestores de topo comunicam os valores fundamentais da incubadora.

2. A força de trabalho da incubadora está ciente dos valores fundamentais.

3. A declaração de missão da incubadora inspira nossa força de trabalho.

4. A declaração de missão da incubadora comunica claramente os valores fundamentais

\section{Sistemas de Restrições}

1. O código de conduta da incubadora informa a nossa força de trabalho sobre os comportamentos que estão fora dos limites.

2. A incubadora tem um sistema que comunica nossa força de trabalho dos riscos que devem ser evitados.

3. Nossa força de trabalho está ciente do código de conduta da incubadora.

4. A incubadora se baseia em um código de conduta para definir o comportamento apropriado da sua força de trabalho.

Indique a intensidade de uso do Sistema de Controle Gerencial e/ou Medidas Administrativas de Controle em âmbitos interno e externo da incubadora. Atribua notas de "1" a "7", sendo: 1 = Não existe na incubadora, 7 = Muito usado de modo geral.

\section{Sistemas de Controle Diagnóstico}

1. Acompanhar o progresso das atividades em direção às metas.

2. Monitorar os resultados.

3. Comparar os resultados com as expectativas.

4. Revisar as medidas chave de desempenho.

\section{Sistema de Controle Interativo}

1. Promover a discussão em reuniões entre superiores, subordinados e pares.

2. Proporcionar o contínuo desafio e discutir os dados subjacentes, os pressupostos e os planos de ação.

3. Fornecer uma visão comum da incubadora.

4. Oportunizar a incubadora concentrar-se em questões comuns.

5. Possibilitar a organização focalizar-se nos fatores críticos de sucesso.

6. Desenvolver um vocabulário comum na incubadora.

Nas assertivas abaixo, aponte o quanto cada condição cultural é incorporada pela incubadora de forma geral e de modo particular para fornecer informações no âmbito interno. Atribua notas de "1" a " 7 ", sendo 1 = pouco presente, 7 = muito presente.

\section{Condições Culturais}

1. Dados históricos (raça, religião, etnia e deslocamento populacional).

2. Ambiente no qual a incubadora se localiza.

3. Variação do gosto e preferências dos clientes.

4. Organizações com capacidade de mudar os valores culturais.

5. Valores e normas que se alteram a medida que os eventos afetam a população envolvida. 